\title{
The Ohio State University Comprehensive Cancer Center
}

National Cancer Institute

\section{Source}

National Cancer Institute. The Ohio State University Comprehensive Cancer Center. NCI Thesaurus. Code C39528.

The Ohio State University Comprehensive Cancer Center strives to eradicate cancer from individuals' lives by creating knowledge and integ rating groundbreaking research with excellence in education and patient-centered care. It was designated as an $\mathrm{NCl}$ comprehensive cancer center in 1976. Patient care is performed at the Arthur G. James Cancer Hospital and Richard J. Solove Research Institute. 